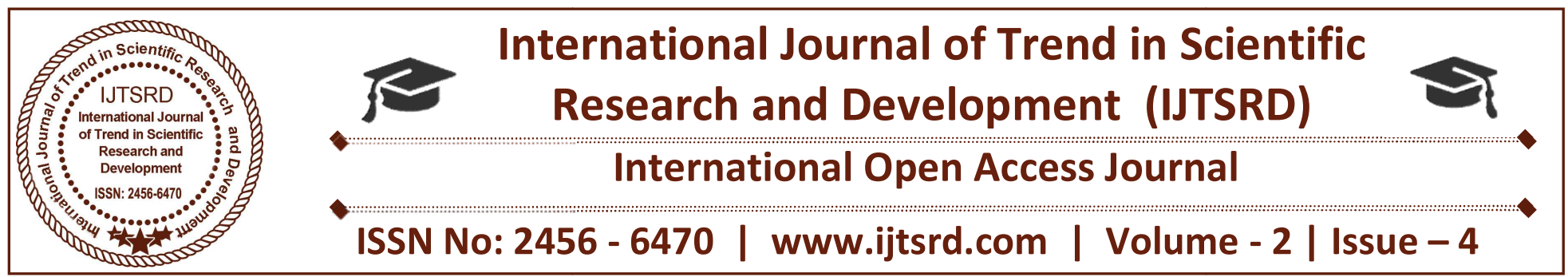

\title{
CDSS implementation with CDA generation and integration for health information exchange in cloud
}

\author{
Pooja N. Umekar, Dr. H R. Deshmukh, Prof. O. A. Jaisinghani, Prof S.V. Khedkar \\ Department of Computer Science \& Engineering, DRGIT\&R, Amravati, Maharashtra, India
}

\section{ABSTRACT}

Electronic health record helps to improve the safety and quality care of every individual patient details, that to be stored in various hospital through health information exchange. The clinical document architecture(CDA) developed by Health level seven(HL7) is core document standard that ensure interoperability of the document. Hospitals are reluctant to adopt interoperable hospital information system due to its deployment cost except for in a handful countries. A problem arises even when more hospitals start using the CDA document format because the data scattered in different documents are hard to manage. CDA document generation and integration Service based on cloud computing through which hospitals are enabled to conveniently generate CDA document per patient into a single CDA document and physician and patients can browse the clinical data in chronological order.

To improve the accuracy and speed of diagnosis, health care system is important to provide the faster and efficient way. A clinical decision support system (CDSS) is a health information technology system that is designed to provide physicians and other health professionals with clinical decision support (CDS), that is assistance with clinical decision -making tasks. The system is designed by using various data mining techniques to assist the diagnosis of patient's symptoms. Our system is designed with the help of Naiive Bayesian classification technique which has overcome the various data mining technique to diagnose the patient symptoms. The Naïve Bayesian classification technique provide the diagnosis of disease with the help of symptoms occurs to the patient ."Clinical decision support systems link health observations with health knowledge to influence health choices by clinicians for improved health. Our system implement (CDSS) clinical decision support system looking towards the system CDSS clinical decision support system diagnose the diseases of the patient and also the CDA is generated which will be in XML form and also it can be integrated through various platforms. With the help of this system the time of patient would be saved and accurate diagnoses of the patient is done.

Keywords: Health information exchange( HL7) CDA, cloud computing, software as a service, CDSS , privacy preserving

\section{INTRODUCTION}

Electronic health record is the systematize collection of patient and population electronically stored information in digital format. These records can be shared across different health care centers, record are shared through network connected, and vast information system or other information network and exchanges. Electronic health records (EHR) it include range of data including static medical history, medication and allergies, immunization status, personal statestics like age and weight, sex [1]. Health Level Seven has established CDA as a major standard for clinical documents. CDA is a document markup standard that specifies the structure and semantics of 'clinical documents' for the purpose of exchange. The first version of CDA was developed in 2001 [2]. Many projects generating CDA have been entirely used in many countries. Active works are 
being done on improving interoperability based on open Electronic Health Records.

Our system consist of CDA generation with implementation of CDSS with the information exchange in cloud, basically the system will generate CDA with the help of patient's information provided to the system, the CDA document consist of the general information provided by patient like his personal details and also the and then the CDSS is generated and when the sharing of CDA takes place privacy is preserved by encrypting the contact number of the patient so we can say that if any of the patient is suffering from disease first the patient is not carring the previous records of health issues so the doctor will be able to see the previous history and with the help of that doctor will be able to select the symptoms and give the prescription to patient.

\section{LITERATURE REVIEW}

Health Level Seven has established CDA as a major standard for clinical documents [3]. CDA is a document markup standard that specifies the structure and semantics of 'clinical documents' for the purpose of exchange. The first version of CDA was developed in 2001 and came out in 2005 [2]. Many projects adopting CDA have been successfully completed in many countries [4]. Active works are being done on improving semantic interoperability based on open Electronic Health Record [7]. To establish confidence in Health Information Exchange interoperability, more Health Information Services's need to support CDA. However, the structure of CDA is complicated and the production of correct Clinical Document Architecture document is hard to achieve without understanding of the CDA sufficient experience with it. In addition, development the Health information system for hospitals vary so greatly that generation of CDA documents in hospital invariably requires a separate CDA generation system. The, hospitals are very reluctant to adopt a new system unless it is absolutely necessary for provision of care. As it is clear that the result, the adoption rate of EHR is very low except for in a few countries such as New Zealand or Australia [9]. In the few years the XML has became the important standard for exchange of information in health care services. When a patient is diagnosed at a clinic, a CDA document record is generated. The CDA document can be shared with other clinics if the patient agrees. The concept of family doctor does not exist in some countries, hence it is common for a patient to visit a number of different clinics.

The exchange process of CDA document is in the following cases: when a doctor needs to study a patient's medical history; when referral and reply letters are drafted for a patient cared by multiple clinics; when a patient is in emergency and the medical history needs to be reviewed. It takes increasing amount of time for the medical personnel record as the amount of exchanged CDA document increases because more documents means that data are distributed in different documents. This significantly delays the medical personnel in making decisions. Hence, when all of the CDA documents are integrated into a single document, the medical personnel is empowered to review the patient's clinical history conveniently in chronological order. In general, hospitals information system are operated independently of each other. We propose a next generation hospital information system (HIS) based on HL7, clinical document architecture (CDA) including also an electronic health record and a clinical data repository (CDR) to enable the data sharing of medical information among medical and health information among health and medical institutions. We designed an XML schema through which an effective clinical document was generated from the HIS after defining the item regulations and the templates.

\section{PROPOSED WORK}

The system is going to perform follows.

- The CDA document is generated in XML format.

- The CDA will consist of the information of the patient the information consist of the name , patient id, symptoms etc

- The CDA will be generated on two platforms, the information will be integrated from one to other platform.

- The main purpose of our system is to implement CDSS with generation of CDA and integrating the health information exchange.

- The system is going to give or create the cloud atmosphere. 
CDA location Data items

\section{CDA HEADER: Document}

Information (creation time, template ID, code, purpose) Patient's information (ID, name, information (ID, name, represented organization) Organization's information (name, address, phone number)

CDA Body: Payers Advance

Directives Support Functional Status Problems Family History Social History Allergies Medications Symptoms Procedures Encounters Plan of Care

TABLE 1: CDA DOCUMENT:

\section{CDSS IMPLEMENTATION}

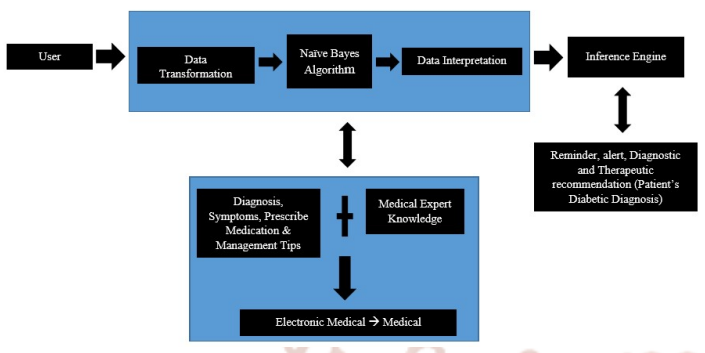

Fig 4.1 cdss system working

The above diagram shows the overall architecture of how CDSS it shows the actual working.

1. CDA generation generates CDA documents. .

2. CDA generation Interface uses the API provided by the cloud and relays the input data and receives CDA documents generated on cloud .

3. Template manager is responsible for managing the CDA documents generated in cloud server.

4. CDA generator collects patients data from hospital and generates CDA documents in template.

5. CDA validator inspects whether the generated CDA documents complies with a CDA document complies with a CDA schema standards.
6. The CDSS is generated, when we give the symtomps to the systems in CDSS the disease is diagnose.

The following steps takes place in CDSS,

1: Doctor Register with the System.

2: Doctor has to login the system with his authentic email-id and password.

3: Doctor can add / edit / update /delete any number of disease, their symptoms, and their prescription information.

4: Doctor add patient information along with the symptoms he is suffering from to the database and check for diagnosis.

5: Using Database will provide the historical medical data present in our database and processing with the help of Naïve Bayesian classifier fuzzywuzzy search algorithm.

6: After calculation, the predicted result will be send to the next level. On this level the probability of predicted disease risk will be calculated and top three disease having probability of more than $50 \%$ are displayed. In this algorithm the maximum probability disease risk will be calculated.

7: Now doctor check the patient symptoms once again and from the result generated in step 6 , he suggest most suitable prescription for patient. Finally, proper predicted diseases will be diagnose, this will help to give proper prescription to the patients more effectively.

8: For more proper CDSS designing, doctor review his prescription suggested to the patient. Here he checks that, the patient gets cure form his provided prescription or not. If the patient gets cure then go to step 9 or stop otherwise.

9: Check for any new symptoms that the patient is suffering from and already our CDSS data have. If any new symptoms are identified, Retrain symptoms to the particular database by adding symptoms to particular disease For example: The CDSS is mainly used to diagnose the disease the symptoms are provided in the database and with the help of those symptoms, the diseases are being diagnose with the help of naviebayes algorithm. 


\subsection{Work flow diagram of CDSS}

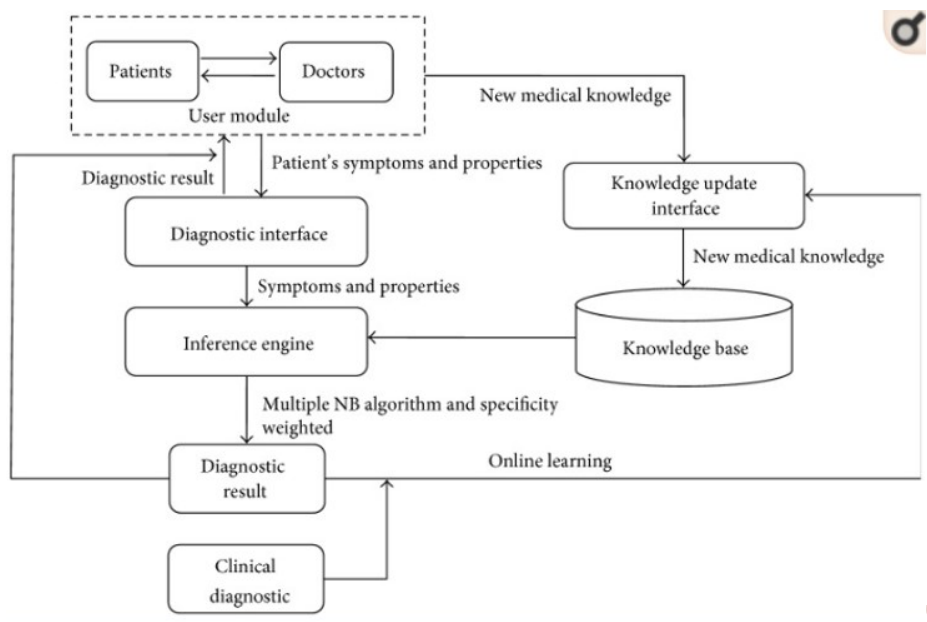

Fig 4.1: the fig shows the how actually the cdss works.

\section{ADVANTAGES:}

- Construction of a cloud computing environment and deployment of CDA generation and integration system based on it.

- Generation of CDA documents on different developer platforms through Cloud.

- CDSS have number of quality improvement benefits, including health care quality and enchanced patient outcomes.

- Avoidance of errors and adverse events

- Improves cost benefits and provider and patient satisfaction.

\section{LIMITATIONS:}

- As a number of HIE based on CDA documents increases, interoperability is achieved, but it also brings a problem while managing various CDA documents per patient becomes inconvenient as the clinical information for each patient is scattered in different documents .

- The following problems were encountered while developing our CDA document generation and integration system. First, the default language of the Amazon Cloud OS is US English and it did not adequately handle Korean language.

\section{CONCLUSION:}

In this paper interoperability between hospitals not only helps improves patient safety and quality of service.

It reduces time and resources spent on data format conversion.

It avoids errors, it will show the correct information of the patient which is saved.

\section{REFERENCES:}

1. sung -hung lee, Jounhyunsmg , and konkim "CDA generation and integration for health information exchange based on cloud computing systems "vol no 2 ,march / april 2016.

2. R. H. Dolin, L. Alschuler, S. Boyer, C. Beebe, F. M. Behlen, P. V.Biron, and A. Shabo, "The HL7 Clinical Document Architecture," J. Am. Med. Inform. Assoc., vol. 13, no. 1, pp. 30-39, 2006.

3. R. H. Dolin, L. Alschuler, C. Beebe, P. V. Biron, S. L. Boyer, D. Essin, E. Kimber, T. Lincoln, and J. E. Mattison, "The HL7 Clinical Document Architecture,"J. Am. Med. Inform. Assoc., vol. 8, pp. 552-569, 2001

4. M. L. M€uller, F. Ückert, and T. B€urkle, "Crossinstitutional data exchange using the clinical document architecture (CDA)," Int. J. Med. Inform., vol. 74, pp. 245-256, 2005.

5. H. Yong, G. Jinqiu, and Y. Ohta, "A prototype model using clinical document architecture (cda) with a japanese local standard: designing and implementing a referral letter system," ActaMedOkayama, vol. 62, pp. 15-20, 2008..

6. K. Huang, S. Hsieh, Y. Chang, F. Lai, S. Hsieh, and H. Lee, "Application of portable cda for secure clinical-documentexchange," J. Med. Syst., vol. 34, no. 4, pp. 531-539, 2010

7. C. Mart_nez-Costa, M. Men_arguez-Tortosa, and J. Tom_asFern_andez-Breis, "An approach for the semantic interoperability of ISOEN 13606 and OpenEHR archetypes," J. Biomed.Inform., vol. 43,no. 5, pp. 736-746, Oct. 2010.

8. MR. Santos, MP. Bax, and D. Kalra, "Building a logical HER architecture based on ISO 13606 standard and semantic webtechnologies," Studies Health Technol. Informat., vol. 160, pp. 161-165, 2010

9. K. Ashish, D. Doolan, D. Grandt, T. Scott, and D.W. Bates, "Theuse of health information technology in seven nations, "Int. J. Med. Informat., vol. 77, no. 12, pp. 848-854, 2008 